\title{
Asymptotic Level Spacing Of The Laguerre Ensemble: A Coulomb Fluid Approach
}

\author{
Y. Chen and S. M. Manning \\ Department of Mathematics \\ Imperial College \\ London SW7 2BZ \\ U. K.
}

October 4, 2018

\begin{abstract}
We determine the asymptotic level spacing distribution for the Laguerre Ensemble in a single scaled interval, $(0, s)$, containing no levels, $E_{\beta}(0, s)$, via Dyson's Coulomb Fluid approach. For the $\alpha=0$ Unitary-Laguerre Ensemble, we recover the exact spacing distribution found by both Edelman and Forrester, while for $\alpha \neq 0$, the leading terms of $E_{2}(0, s)$, found by Tracy and Widom, are reproduced without the use of the Bessel kernel and the associated Painlevé transcendent. In the same approximation, the next leading term, due to a "finite temperature" perturbation $(\beta \neq 2)$, is found.
\end{abstract}




\section{Introduction}

The probability that there are no levels in a scaled interval $(-t, t)$ [where $t$ is measured with respect to the averaged spacing], $E(0, t)$, in a long stack of energy levels of heavy nuclei was given by a conjecture of Wigner[1] and was well supported by experimental data[2] for systems with time reversal symmetry. In a series of seminal papers, Dyson [3] introduced a new class of random matrix ensembles and determined in the continuum approximation (expected to be valid when number of levels, $N$, is very large) that $\ln E_{\beta}(0, t) \sim-\frac{\pi^{2}}{4} \beta t^{2}-(1-\beta / 2) \pi t$, using the methods of classical electrostatics, potential theory and thermodynamics, for ensembles with unitary $(\beta=2)$, orthogonal $(\beta=1)$ and symplectic $(\beta=4)$ symmetries. A term of $O(\ln t)$ and a constant missed in the continuum approximation was later discovered by Widom, des Cloizeaux and Mehta, and Dyson [4, 5, 6, 7, 8].

Recently, in a remarkable paper, Tracy and Widom 9] showed, in the single interval case, that the logarithmic derivative of the Fredholm determinant of the Bessel Kernel—which arises in the scaling limit [with respect to the hard edge density, see [9, 10]] of the unitary Laguerre ensemble - satisfies a Painlevé V equation, from which the asymptotic level spacing distribution can be computed exactly, amongst other quantities of interest from the random matrix point of view 11$]$.

In this letter we shall employ the continuum approximation of Dyson to calculate the level spacing distribution for the Laguerre ensemble [with $\alpha \neq 0$, see 9]], with $\beta=2$. For $\beta \neq 2$, the spacing distribution can be found by a perturbative calculation due to Dyson [3].

From the Brownian motion model, in the "hydrodynamical" approximation, Dyson 12 
derived an equation satisfied by the non-equilibrium level density $\sigma(x, \tau)$;

$$
\frac{\partial}{\partial \tau} \sigma(x, \tau)=\frac{\partial}{\partial x}\left(\beta \sigma(x, \tau) \frac{\partial \Psi}{\partial x}\right), \quad \tau>0
$$

with

$$
\Psi(x, \tau)=\frac{1}{\beta} u(x)-\int d y \sigma(y, \tau) \ln |x-y|+\left(\frac{1}{\beta}-\frac{1}{2}\right) \ln [\sigma(x, \tau)]
$$

where the fictitious time $\tau$ pulls the levels towards the observed level density, $r(x)$, generated by the imposed potential $u(x) / \beta=\int d y r(x) \ln |x-y|,($ as $\tau \rightarrow \infty)$, that holds the Coulomb Fluid together. The stationary solution, reached as $\tau \rightarrow \infty$, for the level density statisfies the Hükel-like self-consistent equation

$$
u(x)-\beta \int d y \sigma(y) \ln |x-y|+\left(1-\frac{\beta}{2}\right) \ln [\sigma(x)]=A=\text { constant }
$$

with effective temperatute $T_{e} \propto\left(1-\frac{\beta}{2}\right)$. This, in turn, maybe derived from the following variational principle: $\min _{\sigma, \mu} F[\sigma, \mu]$, with

$$
F[\sigma, \mu]=\beta \int d x \Psi(x) \sigma(x)-\mu\left(\int d x \sigma(x)-N\right)
$$

and $A=-\mu+(1-\beta / 2)$, where $\mu$ is the chemical potential. Therefore the free energy, $F$, at equilibrium, with exactly $N$ levels contained in an interval $I$ is

$$
F[I]=\frac{1}{2} A N+\frac{1}{2} \int_{I} d x u(x) \sigma(x)-\frac{1}{2}\left(1-\frac{\beta}{2}\right) \int_{I} d x \sigma(x) \ln [\sigma(x)]
$$

subject to $\int_{I} d x \sigma(x)=N$. For the Laguerre Ensemble, $u(x)=x-\alpha \ln x, x \in(0, \infty)$, but as we are using the continuum approximation, an upper band edge, $b \in(0, \infty)$ must be imposed on the level density to produce a finite number of levels. In the large $N$ limit, the level density is $\sigma(x)=\frac{1}{4 \pi} \sqrt{\frac{4 N-x}{x}}, x \in(0,4 N)$ for the unitary case [13, 14]. This is distinct from the Wigner semi-circle law, where $u(x)=x^{2}, x \in(-\infty,+\infty)$. 


\section{Asymptotic Spacing}

The probability distribution that an interval $(0, a)$ contains no levels is, by definition, the ratio of the partition function where all $N$ levels reside in the complement of $(0, a)$ i.e. $(a, b)$ [where $b$ is the upper band edge and $0<a<b]$ to that for which all $N$ levels reside in the full interval i.e. $(0, b)$, and is [3],

$$
\ln E_{\beta}(0, a)=-[F(a, b)-F(0, b)]
$$

In the continuum approximation, $F$ is given by Eq.(5), where $\sigma(x)$ solves Eq. (3) [15]. From thermodynamic considerations, since $F(a, b)$ is the Free Energy in a "constricted" region, we must have $F(a, b)>F(0, b)$. We now proceed to solve Eq. (3) for $\beta=2$, in the interval $(a, b)$. To simplify the mathematics, Eq.(3) is converted into a singular intergral equation by taking a derivative with respect to $x$, and can be solved by a standard $\operatorname{method}[16]$. The solution of this equation is

$$
\sigma(x)=\frac{1}{\pi^{2} \beta} \sqrt{\frac{b-x}{x-a}} \int_{a}^{b} \frac{d y}{y-x} \sqrt{\frac{y-a}{b-y}}\left(1-\frac{\alpha}{y}\right)=\frac{1}{\pi \beta} \sqrt{\frac{b-x}{x-a}}\left(1-\frac{\alpha}{x} \sqrt{\frac{a}{b}}\right)
$$

where $x \in(a, b)$ and to maintain positivity we demand that $\sqrt{a b}>\alpha$. A straightforward calculation supplies the normalization condition:

$$
N=\frac{b-a}{2 \beta}+\frac{\alpha}{\beta}\left[\sqrt{\frac{a}{b}}-1\right]
$$

We have deliberately left $\beta$ without setting it equal to 2 in Eqs. (7) and (8). It is clear from the structure of Eq.(8), that $N$, the total number of levels, is almost exhausted by the first term, but as to be seen later, the second term cannot be discarded. As we are required to compare $F(a, b)$ with $F(0, b)$, where $N$ is very large, to facilitate the computation we shall

evaluate instead, $F(a, b)$ in the limits $a \ll b, \frac{a}{b-a} \ll 1$ and $\frac{b}{b-a} \sim 0(1)$, thus by-passing 
an independent computation of $F(0, b)$. To evaluate $F(a, b)$ requires the determination of $A$ and the "interaction energy" $\frac{1}{2} \int_{a}^{b} d x \sigma(x) u(x)$. In the limits stated we shall extract the very large terms, which are functions of $N$ only and finite terms that are functions of $N a$ only; any remaining terms are therefore negligable in the large $N$ limit. Note that the very large terms are then subtracted according to the definition of $E_{\beta}(0, a)$, leaving behind terms only those terms in $N a$.

For the constant $A$, we send $s \rightarrow b^{-}$in Eq.(3) [with $\beta=2$ ], which gives

$$
A=a-\frac{b-a}{2} \ln \left[\frac{b-a}{4 e}\right]-\alpha \ln b-\alpha\left[\sqrt{\frac{a}{b}}-1\right] \ln (b-a)+\frac{\alpha}{\pi} \sqrt{\frac{a}{b}} I\left(\frac{a}{b-a}\right),
$$

where

$$
\begin{aligned}
I(x) & :=\int_{0}^{1} d t \sqrt{\frac{1-t}{t}} \frac{\ln (1-t)}{t+x}=\left.\frac{1}{x} \frac{\partial}{\partial \beta}\left[B\left(\frac{1}{2}, \beta\right) F\left(1, \frac{1}{2}, \frac{1}{2}+\beta,-\frac{1}{x}\right)\right]\right|_{\beta=3 / 2} \\
& =\sim-2 \pi(1-\ln 2)+\pi \sqrt{x}+\cdots, x \ll 1 .
\end{aligned}
$$

As $N \rightarrow \infty$, but with $N a$ finite, we find that

$$
\frac{1}{2} N A \sim-N^{2} \ln (N / e)-\frac{\alpha}{2} \ln \left(16 N / e^{4}\right)+\frac{1}{2} N a-\frac{\alpha}{2} \sqrt{N a},
$$

where to capture all the terms in $N a$ it is essential to use Eq.(8) for $N$. For the contribution due to the interaction, we have (for $\beta=2$ )

$$
\begin{gathered}
\frac{1}{2} \int_{a}^{b} d t \sigma(t) u(t)=\frac{1}{2}\left[\frac{(b-a)}{2 \beta}\right]^{2}+\frac{1}{2} a \frac{(b-a)}{2 \beta}-\frac{\alpha}{2} \frac{(b-a)}{2 \beta}\left[\sqrt{\frac{a}{b}}-1\right] \\
-\frac{\alpha}{\pi} \frac{(b-a)}{2 \beta} h\left(\frac{a}{b-a}\right)-\frac{\alpha^{2}}{2 \beta} \ln (b-a)\left[\sqrt{\frac{a}{b}}-1\right]+\frac{\alpha^{2}}{2 \beta \pi} \sqrt{\frac{a}{b}} g\left(\frac{a}{b-a}\right),
\end{gathered}
$$

where

$$
h(x):=\int_{0}^{1} d t \sqrt{\frac{1-t}{t}} \ln (t+x) \sim-\frac{\pi}{2} \ln (4 e)-\pi x+2 \pi \sqrt{x}+\cdots, \quad x \ll 1
$$


and

$$
\begin{aligned}
g(x) & :=\int_{0}^{1} \frac{d t}{t+x} \sqrt{\frac{1-t}{t}} \ln (t+x) \\
& =-\left.\frac{\pi}{2} \frac{\partial}{\partial \rho}\left[x^{-\rho} F\left(\rho, \frac{1}{2} ; 2 ;-1 / x\right)\right]\right|_{\rho=1} \sim \frac{\pi}{\sqrt{x}} \ln (4 x)+\pi \ln x+\cdots, x \ll 1
\end{aligned}
$$

With the same considerations,

$$
\frac{1}{2} \int_{a}^{b} d t \sigma(t) u(t) \sim \frac{1}{2} N^{2}-\alpha N \ln \left(2 / e^{1 / 2}\right)-\frac{\alpha^{2}}{2} \ln 4 N+\frac{1}{2} N a-\frac{3}{2} \alpha \sqrt{N a}+\frac{\alpha^{2}}{4} \ln (4 N a) .
$$

Pooling together Eqs.(11) and (15) we find by subtracting the very large terms

$$
F(a, b)-F(a \ll b, b) \sim N a-2 \alpha \sqrt{N a}+\frac{\alpha^{2}}{4} \ln (4 N a)
$$

which gives,

$$
E_{2}(0, s) \sim \frac{\mathrm{e}^{-s / 4+\alpha \sqrt{s}}}{s^{\alpha^{2} / 4}}
$$

upon scaling with respect to the hard edge density, i.e. with the replacement $N a \rightarrow \frac{s}{4}$.

Before we proceed to give the result for the $\beta \neq 2$ case, we should like to mention that by repeating the calculation in the far simpler situation where $\alpha=0$, Eq.(8) can be solved trivially, which gives $b-a=4 N$ [for $\beta=2$ ] and the change in the free energy is equal to $N a$ or $\frac{s}{4}$. This is the exact result of Edelman 17 and of Forrester 18; $E_{2}(0, s)=\mathrm{e}^{-s / 4}, \alpha=0, s \in(0, \infty)$. For $\alpha \neq 0, \beta=2$, Tracy and Widom [9], found through an asymptotic expansion of a Painlevé V equation, higher order terms, in $1 / \sqrt{s}$, $1 / s$ etc., and made a conjecture concerning the term independent of $s$. It was observed by Tracy and Widom that [9] with $\alpha=\mp \frac{1}{2}$, the Bessel kernel reduces to the kernels which arise by scaling into the bulk of the spectrum of the Gaussian orthogonal and symplectic ensembles, respectively, provided one makes the replacement $s \rightarrow \pi^{2} t^{2}$. It is amusing 
to see that by approching the GOE and GUE through the "back door", i.e. using the mapping of Tracy and Widom[9]; the Coulomb Fluid approach, when applied to the Laguerre ensemble, supplies rather precise information 19.

\section{Correction to the Free Energy When $\beta \neq 2$}

This is simply found by adding the finite temperature contribution

$$
\frac{\delta F(a, b)}{\left(1-\frac{\beta}{2}\right)}=\int_{a}^{b} d x \sigma(x) \ln [\sigma(x)] \sim-\ln \left[\frac{2 \pi}{e}\right] N-\frac{\alpha}{2} \ln 4 N+\frac{\alpha}{4} \ln 4 N a, \quad a \ll b-a,
$$

to the $\beta=2$ Free Energy.

Collecting the appropriate terms together, we find that

$$
-\ln E_{\alpha}(0, s) \sim \frac{s}{2 \beta}-\frac{2 \alpha}{\beta} \sqrt{s}+\frac{\alpha^{2}}{2 \beta} \ln s+\left(1-\frac{\beta}{2}\right) \frac{\alpha}{4} \ln s,
$$

which clearly reduces to Eq.(17) when $\beta=2$.

\section{Conclusion}

We should like to mention that by treating the constraint, i.e. Eq.(\$) more accurately, it should be possible to produce the higher order correction terms $1 / \sqrt{s}, 1 / s \ldots$ mentioned previously. However, the computation would become exceedingly complicated and clearly the methods of Tracy and Widom 9 have much to be desired.

The above calculations suggest upon comparison with exact results that the Coulomb Fluid approach is quite robust and may shed light on the level spacing distribution of the $q$-Laguerre ensemble, which arises in the context of electronic transport in disordered systems [20], with the potential

$$
u(x, q)=\sum_{n=0}^{\infty} \ln \left[1+(1-q) x q^{n}\right], \quad q \in(0,1)
$$

which reduces to the ordinary Laguerre potential as $q \rightarrow 1^{-} ; u\left(x, 1^{-}\right)=x$. We leave as a future project the determination of the level spacing distribution with $u(x ; q)$. 


\section{References}

[1] E. P. Wigner, Gatlinberg Conference on Neuron Physics, Oak Ridge National Laboratory Report ORNL 2309 (1957) 59.

[2] N. Rosenzweig and C. E. Porter, Phys. Rev. 120 (1960) 1698; Statistical theories of spectra:fluctuations, Ed. C. E. Porter, Academic Press (New York) 1965.

[3] F. J. Dyson, J. Math. Phys. 3 (1962) 140, ibid., 3 (1962) 157, ibid., 3 (1962) 166. See especially the second paper, where the asymptotic level spacing distribution was determined.

[4] H. Widom, Indiana Univ. Math. J. 21 (1971) 277.

[5] J. des Cloizeaux and M. L. Mehta, J. Math. Phys. 14 (1973) 1648.

[6] F. J. Dyson, Comm. Math. Phys. 47 (1976) 171.

[7] In an application of the continuum approximation, the quantity $r_{\beta}(n, t):=\frac{E_{\beta}(n, t)}{E_{\beta}(0, t)}$ was calculated, where $E_{\beta}(n, t)$ is the probability that there exactly $n$ levels contained in the interval $(-t, t)$, with $1 \ll n \ll t$. See, F. J. Dyson, The Coulomb fluid and the fifth Painlevé transcendent IASSNSS-HEP-92/43 preprint, to appear in the proceedings of a conference in honour of C. N. Yang, ed. S.-T. Yau. $r_{\beta}(n, t)$ is also computed in E. L. Basor, C. A. Tracy and H. Widom, Phys. Rev. Lett., 69 (1992) 5.

[8] As will be seen later, the computation of $E_{\beta}(0, t)$, involves subtraction of two large terms which are functions of $N$ and the continuum approximation is not expected to have sufficiently fine resolution to determine the constant. 
[9] C. A. Tracy and H. Widom, Level spacing distributions and the Bessel Kernel, ITD 92/93-7 preprint to appear in Comm. Math. Phys.

[10] By scaling into the bulk and the "soft edge" of the spectrum one gets the sine and Airy kernels respectively, see C. A. Tracy and H. Widom, Fredholm Determinants, Differential Equations and Matrix Models, ITD 92/93-17 preprint to appeared in Comm. Math. Phys.

[11] The Painlevé V in [9] is reducible to a Painlevé III.

[12] F. J. Dyson, J. Math. Phys. 13 (1972) 90.

[13] B. V. Bronk, J. Math. Phys 6 (1965) 228.

[14] T. Nagao and M. Wadati, J. Phys. Soc. Japan 60 (1991) 3298.

[15] For a discussion on the continuum approximation, the reader is urged to consult the second paper of [3].

[16] N. I. Akhiezer and I. M. Glazman, Theory of Linear Operators in Hilbert Space,pg. 114-115, vol. 1, Trans. Merlynd Nestell, Federick Ungar, New York (1961).

[17] A. Edelman, SIAM J. Matrix. Anal. Appl. 9 (1988) 543; Linear Alg. and its Appl. 159 (1991) 55.

[18] P. J. Forrester, Nucl. Phys. B402 [FS] (1993) 709.

[19] The authors should like to thank Craig Tracy for bringing out this point. Specifically, $\ln E_{2}^{\alpha=\mp 1 / 2}\left(0, \pi^{2} t^{2}\right)=\ln D_{ \pm}(t) \sim-\pi^{2} t^{2} / 4 \mp \pi t / 2-\frac{1}{8} \ln (\pi t)$. The quantities $D_{ \pm}(t)$ can 
be found in chapter 12 of M. L. Mehta, Random Matrices, $2^{\text {nd }}$ Edition, Academic Press (New York) 1991.

[20] These types of ensembles are related to $q$-orthogonal polynomials associated with indeterminate classical moment problems, where the potential, $u(x) \sim[\ln x]^{2}$, for very large $x$, is marginally confining and has application to transport in disordered systems. See Y. Chen, M. E. H. Ismail and K. A. Muttalib, J. Phys. Condens. Matter 5 (1993) 177. It can be shown that the level density at the origin, $\sigma_{N}(0, q)$, is $(1-$ $\left.q^{N}\right) / \ln (1 / q)$. 